turn inwards until they cross one another. In diagnosis lumbar puncture is invaluable and free from risk if done with ordinary care. The presence of the specific micro-organisms can thus often be determined and a cytological examination of the centrifugalised fluid made. Too much stress must not, however, be laid on the fact, useful for differential diagnosis, that in the epidemic form the polymorphonuclear neutrophiles are immensely increased, while in meningitis associated with tubercle the exudate consists almost entirely of lymphocytes, for in the later stages of the illness lymphocytes may almost entirely replace the multinuclear leucocytes so abundant in the earlier effusion. Netter lays more stress for diagnosis on the macroscopic appearance of the lymph clot, which in the epidemic disease is yellowish, soft, and readily spread out, while in tubercular meningitis it is white, resistant, and spreads out with difficulty. Although well established as a safe and certain diagnostic measure, the therapeutic worth of lumbar puncture is less recognised and as a method of cure it is still on its trial. There is, however, an increasing inclination to regard it as a hopeful procedure. Simple withdrawal of the fluid is more often practised, but in some cases the injection of antiseptic solutions into the spinal canal after withdrawal is attempted and with some measure of success. Lysol in one per cent. solution is a favourite application and about 10 cc. may be injected at once. Lenhartz ${ }^{10}$ advocates early and repeated puncture, and claims success for the treatment. He withdraws from 30 to $50 \mathrm{cc}$, and repeats the process as often as pressure symptoms recur. The best spot for puncture is the junction of a perpendicular dropped from the iliac crest with the line of the spine, the patient lying on one side with the thighs strongly flexed. The fluid should always be withdrawn slowly and it is advisable to measure the intraspinal pressure. Relief of pressure symptoms for the time almost always follows, even in cases not permanently benefited. The withdrawal of purulent fluid removes a large number of pathogenic organisms and unless the foramen of Magendie is closed it also relieves ventricular dropsy, and hence may lessen the occurrence of a serious sequela-chronic hydrocephalus. Certainly where the pus is present under considerable tension it seems reasonable to expect a relief of constitutional and local symptoms such as one observes on the evacuation of pus from other regions of the body, as e.g. from a subcutaneous abscess. The application of hydrotherapy was introduced by Aufrecht in 1894 and has received the approval of the majority of those who have employed it, but the method of application has varied considerably. Thus Aufrecht gave only one bath daily, while Barr employed a prolonged immersion of eleven days or more. Netter, who has had extensive experience of, and warmly recommends, the treatment, advises a hot bath lasting from half to three-quarters of an hour, repeated four or five times a day. Recent and enthusiastic testimony as to the benefit of this treatment comes from Russia, where Rojansky ${ }^{11}$ has employed it for five years in the treatment of 51 patients in his women's wards. His method is to give a bath at a temperature of $104^{\circ} \mathrm{F}$. for 15 or
20 minutes once or twice a day, applying an ice cap to the head at the same time. Pain, restlessness, delirium, and unconsciousness are rapidly and decidedly relieved. Of the cases so treated 17 died, a mortality of 33 per cent. Rojansky's results are a valuable contribution to the effect of the bath treatment, because an accurate control was provided by the simultaneous treatment, without baths, of 50 cases admitted to the male wards, of whom 40 , or 80 per cent., died. An interesting contribution to this subject has been made by the employment, in a few cases, of subcutaneous injections of diphtheria antitoxin. Wolff ${ }^{12}$ of Hartford, Conn., was led to use it in three or four cases, which recovered, as a result of his observation that a pronounced antagonism existed between the diplococcus intracellularis and the Klebs-Loeffler bacillus, and that pure cultures of the former were killed by the addition of antidiphtheritic serum. Large doses, 6,000 to 10,000 units were employed. Waitzfelder, of New York, has tried it also and reports a few striking cases of recovery, but although the treatment seems deserving of further trial no certain conclusions can be deduced from the small number of cases hitherto treated. It is difficult readily to assess the value of any particular form of treatment as epidemics vary much in severity, and while the mortality rarely falls as low as 30 it has reached as high as 77 per cent. Moreover epidemics are usually somewhat equably distributed in the area affected so that it is not the rule for any very large number of patients to come under the treatment of one physician. Hence statistics showing the result of treatment must be accepted with perhaps more than average caution.

Med. Rec., May 6, p. 716. ${ }^{2}$ Lancet, May 6, p. 1214. ${ }^{3}$ Med. Rec., April 15, p. 562. ${ }^{4}$ Ibid, May 6, p. 716. 5 Lancet, April 15. 6 Med. Rec., March 11, p. 365 . 7 Ibid, Feb. 4, p. 195. 8 Ibid May 6. 9 Ibid, April 15. ${ }^{10}$ Lancet, April 15, p. 1011. ${ }^{11}$ Med. Rec., April 1. ${ }^{12}$ Ibid, March 11.

\section{SURGERY OF THE STOMACH.}

Gastric Ulcer.-The surgical treatment of gastric ulcer is one of great importance owing to the disastrous effects which result if the ulcer ruptures and permits the escape of intestinal contents into the general peritoneal cavity. A consideration of such cases suggests many points as referred to by Clarke. ${ }^{1}$ What is the causation of these ulcers? Two main causes have been assigned for the formation of ulcers, namely thrombosis of the vessels of the stomach and the production of an excess of hydrochloric acid during the process of digestion. The difficulty of proof that either of these conditions is the chief factor in the causation of ulceration is, however, very great. One thing is absolutely certain, that whenever these cases have been under skilled observation before perforation has occurred, they are always found to be associated with indigestion, and usually with the presence of bad teeth. Their association with oral sepsis is undoubted, and the men in whom they occur, are generally addicted to drink. It is more than likely that no one cause is responsible for these ulcers. Of more importance to the surgeon is the position of these ulcers. It seems generally 
conceded that nearly all chronic ulcers, whether gastric or duodenal, occur in the neighbourhood of the pylorus; those that perforate are usually found near the lesser curvature, cardiac orifice, or on the back of the organ. Thus although Villard and Pinatelle, ${ }^{2}$ in an analysis of 115 cases, found that gastric ulceration is vary common about the lesser curvature, yet in only one-third of all cases of perforation is the ulcer situated in this region. This is to be accounted for by the high situation of the ulcer, its comparative fixity, its close apposition to other structures, and the frequent formation of adhesions, the latter tending to limit the extravasation and consequent inflammation, which often results in a perigastric abscess. Persistent vomiting is almost always a feature of perforation of an ulcer of the lesser curvature.

As Clarke ${ }^{3}$ points out the symptoms of ruptured gastric ulcer vary considerably. It is very difficult usually to distinguish a gastric from a duodenal perforation before opening the abdomen, but the rapidity with which the symptoms come on after rupture is usually less after duodenal perforation than after gastric, probably because the duodenum contains less than the stomach, and also because duodenal perforations are, as a rule, smaller than gastric ones. The pain, too, is more often localised behind the right rectus in the case of a duodenal ulcer. When the abdomen has been opened for either of these two conditions, the diagnosis, as a rule, presents much less difficulty. The contents of the stomach consist of semi-digested food and are acid, while the contents of the duodenum, as a rule, are limpid, pale, and alkaline, and remind one almost cf the contents of an ovarian cyst. Duodenal contents, when they are extravasated almost invariably find their way down the right side of the mesentery into the right iliac fossa and pelvis, and thus it is that a duodenal perforation is more likely to be confounded with appendicitis than a gastric perforation is. Pain and shock are almost invariably present, but there are other conditions which give rise to both of these symptoms, and they must be excluded. The next point, therefore, is to determine the course and significance of these two symptoms. It will be found that a lack of abdominal movement is rarely absent in these cases. The abdominal movement is nearly always impaired in the region of the liver and stomach, and in ruptured duodenal ulcers more often on the right side of the abdomen than the left. Gentle palpation will reveal a painful area, possibly very limited in extent, and usually just behind one of the recti. The escape of free gas may also give rise to a diminution of the area of liver dulness, but too much reliance must not be placed on this symptom, as it may be simulated by intestine overlapping the liver. Clarke does not think it necessary to attempt to wash out every particle of food after the ulcer has been closed, but considers it a wise precaution to insert a drain for at least 24 hours in some part of the peritoneal cavity. Nothing should be given by the mouth for at least 24 or 48 hours after operation.

Gastro enterostomy in the treatment of gastric ulcer is very valuable both as an indirect means of stopping hæmorrhage and for the cure of chronic ulcers. Connell ${ }^{4}$ gives many reasons why the direct methods (excision of ulcer, ligation of principal artery, cauterisation of ulcer, etc.) of dealing with hæmatemesis from ulceration are impracticable and frequently cannot be carried out. The difficulty of locating the ulcer is often very great, and the source of a hæmorrhage sufficient to cause death may easily escape detection. The fact that in 20 per cent. of the cases the lesion is multiple, the presence of firm and vascular adhesions and the indistinct limitations of the pathological tissues have all tended towards making impracticable the direct methods of attack of the bleeding point. This has been the cause of a general turning to the indirect methods, especially to that apparently all-healing operation, gastroenterostomy. The exact manner in which a gastroenterostomy usually causes a healing of the ulcer is generally explained in the following manner :-The anastomotic opening allows of a comparatively perfect drainage; the stomach is rapidly emptied and is therefore given rest and quiet; the hyperchlorhydria is diminished, and, as the opening is at the most dependent portion of the stomach, and to the left of the ulcer-bearing area, the ulcer is not irritated by prolonged contact with the stomach contents. The chief reason for expecting hæmorrhage to cease after the operation will be on account of the rest given to the stomach by the new opening, so, to a large extent doing away with peristalsis, and at the same time allowing the organ to contract; and also the lack of irritation of the bleedingpoint by the stomach contents. These conditions and others do, without doubt, favour the stoppage of hæmorrhage and the formation of a clot. But that there are cases in which these measures are not sufficient to arrest the hæmorrhage cannot be doubted, and, therefore, as we are not able to determine with any reasonable degree of accuracy as to the nature of the source of the hæmorrhage, it would seem that a gastro-enterostomy is indicated only after a thorough search for the bleeding-point.

Duodenal Ulcer has, perhaps, not received the attention it deserves. It has been stated that all duodenal ulcers are secondary to gastric ulcers, but Mayo ${ }^{5}$ says this has not been borne out by his experience. Considering the known errors and the possibilities springing from a pre-determined gastroenterostomy and imperfect examination of an ulcer situated in the pyloric region, it must be evident that a duodemal ulcer is a far more common condition than has been thought. Mayo had 59 operations in 58 cases. Of these, seven were for acute conditions, developing, with one exception, upon chronic ulcer with three deaths. Fiftyone operations for chronic conditions gave one death. At the present time posterior gastroenterostomy would appear to be the operation of choice in the chronic cases, but the last word has not yet been said. It is fairly certain that even with a large gastro-enterostomy the food will pass out by preference through an unobstructed pylorus by muscular action. Gastro-enterostomy performed for gastric ulcer is open to the same objection if there be no stenosis. For this reason, when the ulcer does not cause at least partial obstruction, it may be necessary to artificially block the pyloric outlet. After gastro-enterostomy the food, as a rule, passes out quickly, but there may be attacks 
of biliary regurgitation at intervals of days or weeks, and it is sometimes necessary to reoperate to check the disturbance. If the patient is in good condition Mayo now performs a posterior suture gastroenterostomy after the clamp method introduced by Moynihan. Four inches below the completed gastro-enterostomy an entero-anastomosis, with suture between the two lines of the bowel, is made, using the holding clamps. To prevent bile arising to the level of the stomach and also to cause the food to always pass out by the efferent bowel, the afferent intestine between the entero-anastomosis and the gastro-enterostomy should be closed by an infolding stitch which turns the periphery of the intestine into the lumen or by other methods.

1 Clin. Jour., Feb. 1, 1905, p. 211. 2 Med. Chron., Oct. 1904, p. 39. 3 Loc. cit. 4 Annals of Surg., Oct. 1904, p. 500. ' 5 Ibid., Dec. 1904 , p. 886 .

\section{SURGERY OF THE VERMIFORM APPENDIX.}

The Treatment of the Stump in Appendicectomy has been the subject of much difference of opinion and many methods of dealing with the stump have been described. Yet Seelig ${ }^{1}$ claims that a careful search of the literature fails to reveal a single article in which the special treatment of the stump is based on definite anatomical, pathological, or experimental data. In general the methods of treating the stump may be grouped under three heads, as follows:-1. Ligation of the base of the appendix, ablation of the organ distally to the ligature, and inversion of the stump by means of Lembert sutures passed through the peritoneum of the cæcum. In such cases the appendix stump is inverted into the wall of the eæcum. 2. Ablation of the organ without previous ligature of its base, and inversion of the stump into the lumen of the cæcum; Lembert sutures over the site of the inversion. '3. Simple ligation, ablation of the organ distally to the ligature, and cauterisation (with pure carbolic acid or actual cautery) of the stump. Seelig endeavours to show that the first and second methods are langerous, and that the third method is not only free from these dangers, but also possesses distinct advantages. In the first method the appendix stump is invaginated into the cæcal wall. It is impossible to invaginate it into the cavity of the cæcum, because the base of the appendix has been ligated preliminary to ablation of the organ. Even if the stump has been cauterised, and thus disinfected, before inversion into the cæcal wall, the method is nevertheless a dangerous and unreliable one; for the inevitable exudate that forms is bottled up in a cavity under conditions which are particularly favourable to abscess formation. Covering the ligated stump with a previously prepared cuff of peritoneum is only a modified way of locking it up in a closed space, and is open to the same objections. In the second method the ixpendix stump is inverted directly into the cæcum. 'There is no danger, therefore, of imprisoning a istump exudate, for drainage into the cæcal pouch is complete. In this method, however, it is imloossible to pass a ligature around the base of the appendix, for such a ligature would prevent complete inversion of the stump, and the result, therefore, is that the field of operation is exposed to infection by its communication with the infected cavity of the fæces-laden cæcum. There is also a danger of secondary hæmorrhage by this method, for the appendicular artery, which runs in the walls of the appendix in a fair proportion of cases, remains unligatured. The third method of simple ligature of the appendix, ablation distally to the ligature and cauterisation of the stump, is a safe and rational method. The objection that this method leaves uncovered, in the free peritoneal cavity, an infected stump is not valid, for it has been shown that when the stump is cauterised either by pure carbolic acid or the actual cautery it is sterile. The argument that an uncovered stump leads to the formation of adhesions which so frequently distress the patient after operation has also been shown to be erroneous. Simple ligation and cauterisation may therefore be said to be absolutely safe. Under certain circumstances the appendix cannot be dealt with in this simple way, and when difficulty is experienced by reason of extensive adhesions of the appendix due to the results of repeated previous attacks of local inflammation, Isaacs ${ }^{2}$ recommends that the method of "decapsulation" as the best means of overcoming the difficulties encountered. Having located some portion of the appendix, preferably the proximal, the capsule of the appendix is incised longitudinally opposite the mesenteric attachment if possible, so as to avoid the larger blood-vessels. The white, glistening surface of the middle layer is the guide to the depth of the incisions. The incised serous coat peels off very easily, and with the handle of the scalpel or with a periosteum elevator, the core can be easily separated and raised from its bed without fear of hæmorrhage. This white core now serves as a guide to the capsule, inside of which it can be loosened for a short distance, then the capsule split for that distance, and the procedure repeated till the whole length is delivered. When the appendix is perforated the inner layers are gangrenous and the core is, therefore, likely to tear within its capsule on the least traction. In such a case, while it makes conditions somewhat more difficult, it is comparatively easy to follow up the capsule, find the other end of the torn core and proceed with the decapsulation. There will frequently be found a point of maximum adhesions, centralised usually at the point of pressure-necrosis from a fæcal concretion, or due to some other cause, and when this is reached and freed by decapsulation, the remainder of the appendix, be it either the proximal or the distal portion, may be found comparatively free and can be dealt with in the usual manner, taking it off together with its capsule after ligation of the mesenteric attachment which, at this stage, can be easily recognised. In the closure of the abdominal wall after appendicectomy, Bell ${ }^{3}$ recommends using as sutures thin strands of the external oblique tendon, which can be easily split off. This certainly gets rid of the vexed question of infection from catgut.

1 Amer. Jour. Med. Sci., Nov. 1904. ${ }^{2}$ Med. Rec., April 15, 1905. 3 Brit. MeJ. Jour., Oct. 29, 1904, p. 1145. 